\title{
BPS Saturated String Amplitudes, K3 Elliptic Genus and Igusa Cusp Form $\chi_{10}$
}

\author{
Stefan Hohenegger* \\ Max-Planck-Institut für Physik \\ Werner-Heisenberg-Institut \\ 80805 München, Germany \\ E-mail: shoheneg@mppmu.mpg.de
}

We discuss the elliptic genus of $\mathrm{K} 3\left(\phi_{K 3}\right)$ from a conformal field theory and an effective action point of view as an example of a BPS saturated quantity in string theory. In the former approach we give evidence for an action of the Mathieu group $\mathbb{M}_{24}$ on the space of BPS states contributing to $\phi_{K 3}$ (Mathieu moonshine). On the effective action side we present a new series of 1/4 BPS saturated one-loop amplitudes in type II string theory on $K 3 \times T^{2}$ whose integrand can be related to derivatives of $\phi_{K 3}$. We evaluate explicitly a generating functional for this class of amplitudes and show that it is captured by the weight ten Igusa cusp form of $\operatorname{Sp}(4, \mathbb{Z})$. We conclude by speculating on possible algebraic implications of these results on the space of BPS states of the $\mathscr{N}=4$ type II compactification.

Proceedings of the Corfu Summer Institute 2011 School and Workshops on Elementary Particle Physics and Gravity

September 4-18, 2011

Corfu, Greece

${ }^{*}$ Speaker. 


\section{BPS Saturated Quantities in String Theory}

During the last decades our understanding of string theory has dramatically improved and a great number of new and unexpected structures has emerged. Such symmetries and dualities in turn have proven to be powerful tools which allowed us to approach interesting physical (and mathematical) questions. Among the few guiding principles in the search of new structures is to study those sectors of the theory which are insensitive to certain deformations or corrections. In particular, supersymmetrically protected objects have been an invaluable source of insights over the years. In string theory we have two natural paths to approach such BPS-saturated quantities, namely either through conformal field theory (world-sheet point of view) or through the effective (space-time) action and its spectrum (target space point of view). In many cases there is an interesting interplay between both approaches, whose combination has led to many interesting complementary insights.

From the target space point of view BPS saturated quantities appear as very particular interaction terms in the effective action of string compactifications with extended supersymmetry. Such couplings usually enjoy interesting analyticity properties depending on the amount of supersymmetry preserved. An important class of examples are chiral couplings in theories with $\mathscr{N}=2$ supersymmetry $^{1}$ (see e.g. [1]), which are constrained to depend holomorphically on the vector multiplet moduli. Similar examples can be formulated in theories with more supersymmetry, in which chirality is generalized to $\mathrm{G}$ (rassmann)-analyticity of (harmonic)superspaces (see e.g. [2]). Couplings of this type usually enjoy so-called non-renormalization theorems, which makes them much simpler to handle from a field theoretic point of view.

From the world-sheet point of view, BPS saturated quantities take the form of certain indexlike structures or (super)traces, which only receive contributions from (a subset of) the BPS states of the theory. The analyticity properties in the target-space approach, which we mentioned above, usually translate into certain differential equations satisfied by these objects. Such equations in many cases turn out to be very useful computational tools, which allow us to elegantly handle BPS saturated quantities. From a mathematical point of view, such objects encode important topological invariants of the internal manifold of the string compactification.

Over the years many explicit examples of BPS saturated objects have been studied in string theory using both world-sheet as well as target-space methods. A selection of such quantities is schematically depicted in figure 1. One of the most important examples in type II string theory compactified on a Calabi-Yau manifold (thus preserving $\mathscr{N}=2$ supersymmetry in four dimensions), is a certain class of chiral F-terms first discussed in [1], which appear at higher string loop level. These couplings are captured by the (higher genus) partition function of the topologically twisted world-sheet theory ( $\mathscr{N}=2$ topological string). Holomorphicity of these couplings can be formulated as a first order differential equation in the vector-multiplet moduli space [3]. ${ }^{2}$ Further examples of BPS-saturated amplitudes and differential equations satisfied by them have been discussed in [5-11]. These objects have been used to e.g. test string-string dualities (see e.g. [12])

\footnotetext{
${ }^{1}$ Throughout these notes we will count supersymmetries in four dimensions.

${ }^{2}$ Incidentally, due to a non-decoupling of BPS-exact (and thus unphysical) states in the world-sheet CFT, this equation is modified by the so-called holomorphic anomaly [3]. The resulting equation in fact takes the form of a recursive relation which allows in certain cases to compute the partition function up to very high genus [4]. This is an example where BPS properties of certain couplings serve as powerful computational tools in the study of string compactifications.
} 


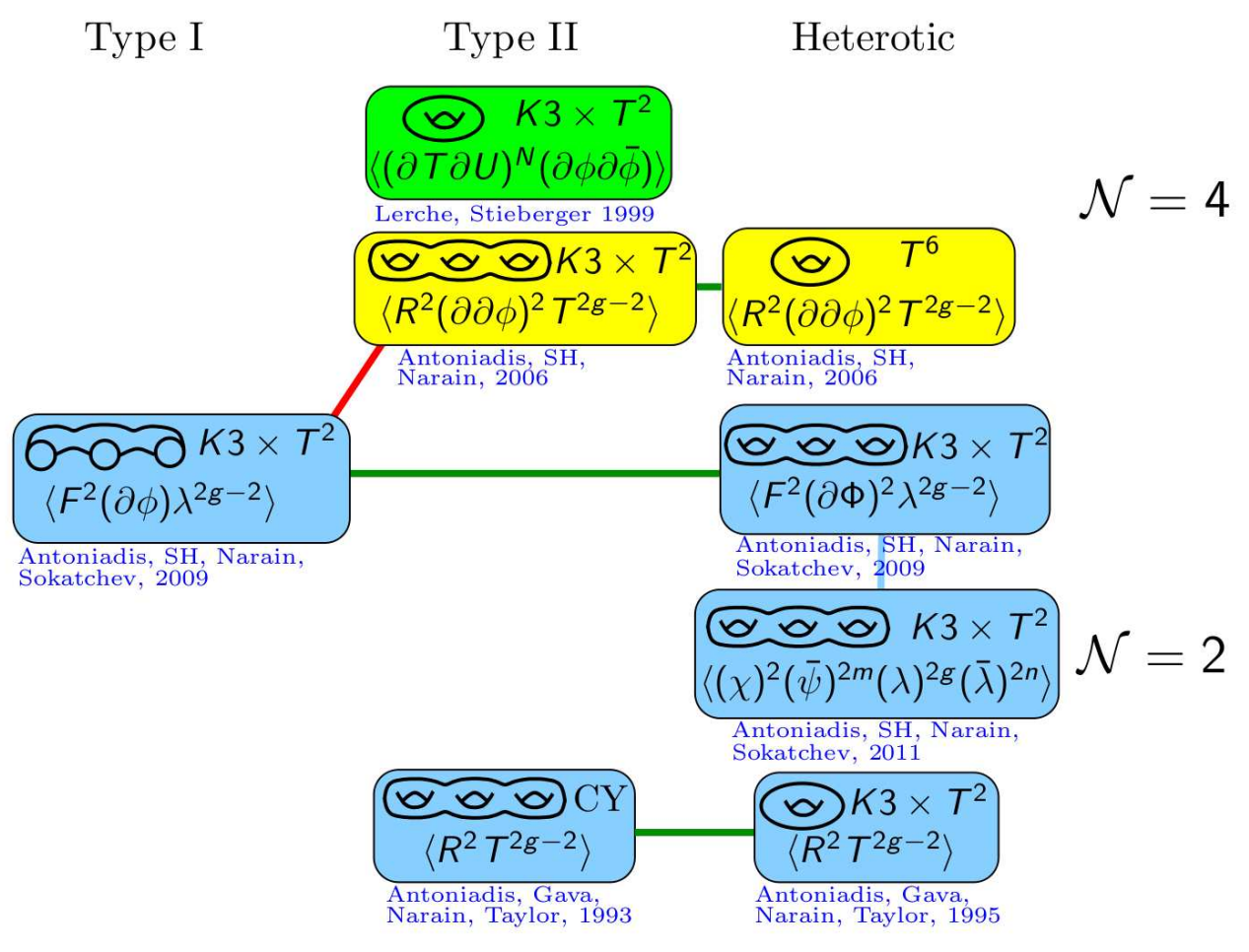

Figure 1: Selection of BPS saturated quantities in string-theory: Different columns correspond to Type I, Type II and Heterotic string theory respectively. Blue and yellow objects stand for $1 / 2$ BPS saturated quantities in $\mathscr{N}=2$ and $\mathscr{N}=4$ compactifications respectively, while the green entry is an example of a $1 / 4$ BPS saturated object in an $\mathscr{N}=4$ theory. Moreover, for each entry, the top right corner specifies the internal manifold of the string compactification, the top left corner indicates whether it is a one-loop or higher loop quantity, while the correlator hints at the explicit physical coupling: Here $R$ denotes the Riemann tensor, $T$ the field strength of the graviphoton, $\phi$ stands for scalar moduli, $\chi, \psi$ and $\lambda$ for fermions of the $\mathscr{N}=2$ hyper- and vector multiplets respectively and finally $F$ the vector multiplet gauge field strength.

or have played an important role in unravelling algebraic structures in the BPS spectrum of string compactifications (see e.g. $[13,14]$ ). The have also turned out to be very important for explicit physical questions, e.g. for the entropy of black holes (see e.g. [15-18]).

In this article - instead of discussing all examples of figure 1 in any detail — we want to focus on one particular 1/4 BPS-saturated quantity and present recent results, both from the target space as well as the world-sheet point of view. The quantity we want to focus on is the elliptic genus of $K 3\left(\phi_{K 3}(\tau, z)\right)$ which is a weak Jacobi form of $S L(2, \mathbb{Z})$ of weight zero and index one. In section 2 we will first take the world-sheet approach and discuss a recent observation of [19]: Upon expansion of $\phi_{K 3}(\tau, z)$ in characters of the $\mathscr{N}=4$ superconformal algebra, the expansion coefficients (i.e. the multiplicities with which each multiplet contributes) of the first few representations are dimensions of irreducible representations of the largest Mathieu group $\mathbb{M}_{24}$. This observation, which is now known under the name Mathieu moonshine, might be understood as a hint towards an $\mathbb{M}_{24}$ symmetry which acts non-trivially on the BPS states that contribute to the elliptic genus 
of $K 3$. We will discuss numerous number theoretic calculations, which give additional weight to this conjecture [20-28]. From, the space-time point of view we will review a new one-parameter family of 1/4 BPS saturated one-loop amplitudes in type II string theory compactified on $K 3 \times T^{2}$. We can define a generating functional for these amplitudes, whose integrand (over the world-sheet torus) is precisely $\phi_{K 3}$. Performing explicitly the loop integration, we can show that this generating functional is related to the logarithm of the Igusa-cusp form, which is the unique weight 10 cusp form of $\operatorname{Sp}(4, \mathbb{Z})$. This results might indicate a connection of $\mathbb{M}_{24}$ with the algebra of BPS states [13] (see also [14] as well as [29, 30, 25, 26]).

\section{World-Sheet BPS Saturated Quantities: The Elliptic Genus of $K 3$}

\subsection{General Properties of the Elliptic Genus}

From the world-sheet perspective, a very important BPS saturated quantity is the elliptic genus of the compactification manifold $\mathscr{M}$. For a world-sheet conformal field theory with central charge $c$ that preserves $\mathscr{N} \geq 2$ supersymmetry, the latter can be defined as the following trace [31-33] in the Ramond-Ramond sector

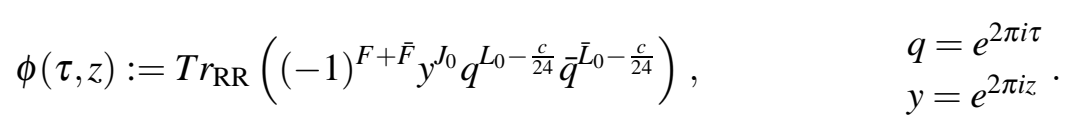

Here $F(\bar{F})$ is the left (right) moving fermion number generator, $J_{0}$ the generator of the left moving $U(1)$ current algebra and $L_{0}\left(\bar{L}_{0}\right)$ a generator of the left (right) moving Virasoro algebra. $\phi(\tau, z)$ is holomorphic in both arguments because only the right-moving ground state can contribute. Moreover, as discussed in [34] the elliptic genus has interesting modular properties: it transforms as a weak Jacobi form of index one and weight zero under $S L(2, \mathbb{Z})$ transformations, i.e. [35]

$$
\begin{aligned}
\phi\left(\frac{a \tau+b}{c \tau+d}, \frac{z}{c \tau+d}\right) & =e^{\frac{2 \pi i c^{2}}{c \tau+d}} \phi(\tau, z), & & \forall\left(\begin{array}{l}
a b \\
c d
\end{array}\right) \in S L(2, \mathbb{Z}), \\
\phi\left(\tau, z+\ell \tau+\ell^{\prime}\right) & =e^{-2 \pi i\left(\ell^{2} \tau+2 \ell z\right)} \phi(\tau, z) & & \forall \ell, \ell^{\prime} \in \mathbb{Z} .
\end{aligned}
$$

The elliptic genus encodes important topological information about the target space manifold $\mathscr{M}$ of the sigma-model. For specific values of the variable $z$ the elliptic genus resembles important signatures and genera, as can be seen from the following table

\begin{tabular}{l|l|l|l}
\hline value & formula & quantity & example $\mathscr{M}=K 3$ \\
\hline$z=0$ & $\phi(\tau, z=0)=\chi$ & Euler number & $\phi_{K 3}(\tau, 0)=24$ \\
$z=\frac{1}{2}$ & $\phi\left(\tau, z=\frac{1}{2}\right)=\sigma+\mathscr{O}(q)$ & $\begin{array}{l}\text { Hirzebruch } \\
\text { signature }\end{array}$ & $\phi_{K 3}\left(\tau, \frac{1}{2}\right)=16+\mathscr{O}(q)$ \\
$z=\frac{\tau+1}{2}$ & $\phi\left(\tau, z=\frac{\tau+1}{2}\right)=\hat{A} q^{-\frac{1}{2}}+\mathscr{O}\left(q^{\frac{1}{2}}\right)$ & $\hat{A}$-genus & $\phi_{K 3}\left(\tau, \frac{\tau+1}{2}\right)=-2 q^{-\frac{1}{2}}+\mathscr{O}\left(q^{\frac{1}{2}}\right)$ \\
\hline
\end{tabular}

Here, as an example, we have presented in the last column the case $\mathscr{M}=K 3$ which gives rise to a world-sheet theory with central charge $c=6$. In fact, all sigma models with $c=6$, which in 
fact preserve $\mathscr{N}=4$ supersymmetry, can be classified through their elliptic genus in the sense that there are only two possibilities

$$
\phi(\tau, z)=\left\{\begin{array}{l}
\phi_{T^{4}}(\tau, z)=0 \\
\phi_{K 3}(\tau, z)=8 \sum_{i=2}^{4} \frac{\theta_{i}(\tau, z)^{2}}{\theta_{i}(\tau, z=0)^{2}}
\end{array}\right.
$$

In the former case, the target space manifold is a four-torus $\mathscr{M}=T^{4}$ while in the latter $\mathscr{M}=K 3$, which has already appeared in the table. Notice, although there is a non-trivial moduli space of $K 3$ surfaces (see equation (3.1) later on), the elliptic genus is the same at every point of $\mathscr{M}_{K 3}$.

\subsection{Expansion in $\mathscr{N}=4$ Characters and Mathieu Moonshine}

As has been discussed in [36] (building on earlier work in [37]), the elliptic genus of $K 3$ (i.e. the non-trivial expression $\phi_{K 3}(\tau, z)$ in (2.4)) can be expanded in a natural manner in elliptic genera of representations of the (left-moving) $\mathscr{N}=4$ superconformal algebra

$$
\phi_{K 3}(\tau, z)=24 \operatorname{ch}_{h=\frac{1}{4}, l=0}^{\mathscr{N}=4}(\tau, z)+\sum_{n=0}^{\infty} A_{n} \operatorname{ch}_{h=n+\frac{1}{4}, l=\frac{1}{2}}^{\mathscr{N}=4}(\tau, z) .
$$

Here we have introduced the elliptic genera of the short $\mathscr{N}=4$ representation

$$
\operatorname{ch}_{h=\frac{1}{4}, l=0}^{\mathscr{N}=4}=\frac{\theta_{1}(\tau, z)^{2}}{\eta(\tau)^{3}} \mu(\tau, z), \quad \text { with } \quad \mu(\tau, z)=i \sum_{n \in \mathbb{Z}} \frac{(-1)^{n} q^{\frac{n(n+1)}{2}} y^{n+\frac{1}{2}}}{\theta_{1}(\tau, z)\left(1-q^{n} y\right)}
$$

with $\mu(\tau, z)$ an Appell-Lerch sum, which is a mock modular theta-function (see [38, 36] and references therein for more information). Besides this, we have also introduced an infinite sequence of elliptic genera of long $\mathscr{N}=4$ representations

$$
\operatorname{ch}_{h, l=\frac{1}{2}}^{\mathscr{N}=4}(\tau, z)=q^{h-\frac{3}{8}} \frac{\theta_{1}(\tau, z)^{2}}{\eta(\tau)^{3}}
$$

The multiplicities, with which each $\mathscr{N}=4$ representation contributes to $\phi_{K 3}(\tau, z)$ are denoted by the coefficients $A_{n}$ in (2.5). Explicitly, the first few of them are

$$
A_{1}=2 \cdot 45, \quad A_{2}=2 \cdot 231, \quad A_{3}=2 \cdot 770, \quad A_{4}=2 \cdot 2277, \quad A_{5}=2 \cdot 5796 .
$$

It was observed in [19] that these numbers are in fact dimensions of irreducible representations of the largest Matthieu group $\mathbb{M}_{24} \cdot{ }^{3}$ This fact is very reminiscent of a similar observation by McKay (and later McKay and Thompson) which lead to developments in mathematics which are today known by the name of 'Monstrous Moonshine' and which are briefly outlined in appendix B (see [39] for a more complete review). Indeed, this analogy suggests that the space of states contributing to $\phi_{K 3}$ takes the form

$$
\mathscr{H}^{\mathrm{BPS}}=\bigoplus_{n}\left(H_{n} \otimes \mathscr{H}_{n}^{\mathscr{N}=4}\right),
$$

\footnotetext{
${ }^{3}$ The next coefficient $A_{6}=2 \cdot 3520+2 \cdot 10395$ itself is not the dimension of an irreducible $\mathbb{M}_{24}$ representation, but can naturally be written as the sum of two $[20,21]$.
} 
with $\mathscr{H}_{n}{ }^{\mathscr{N}}=4$ the space of $\mathscr{N}=4$ representations at mass-level $n$ and $H_{n}$ some representation of $\mathbb{M}_{24}$. A consequence of this would be that the full elliptic genus of $K 3$ can be written in the form

$$
\phi=\left(\operatorname{dim} H_{00}\right) \operatorname{ch}_{h=\frac{1}{4}, l=0}^{\mathscr{N}=4}-\left(\operatorname{dim} H_{0}\right) \operatorname{ch}_{h=\frac{1}{4}, l=\frac{1}{2}}^{\mathscr{N}=4}+\sum_{n=1}^{\infty}\left(\operatorname{dim} H_{n}\right) \operatorname{ch}_{h=n+\frac{1}{4}, l=\frac{1}{2}}^{\mathscr{N}=4}
$$

with $A_{n}=\operatorname{dim}\left(H_{n}\right)$ in equ. (2.5) and the observation of [19] would be tantamount to

$$
\begin{aligned}
& H_{00}=\mathbf{2 3}+\mathbf{1}, \quad H_{0}=\mathbf{1}+\mathbf{1}, \quad H_{1}=\mathbf{4 5}+\overline{\mathbf{4 5}}, \\
& H_{2}=\mathbf{2 3 1}+\overline{\mathbf{2 3 1}}, \quad H_{3}=\mathbf{7 7 0}+\overline{\mathbf{7 7 0}}, \quad H_{4}=\mathbf{2 2 7 7}+\overline{\mathbf{2 2 7 7}} \\
& H_{5}=2 \cdot 5796, \quad H_{6}=2 \cdot 3520+2 \cdot 10395 \text {. }
\end{aligned}
$$

\subsection{Twining Genera}

As a non-trivial check for this 'Mathieu moonshine', it was first proposed in [20, 21] and latter on elaborated in $[22,23]$ to consider so-called twining genera, which are the analogue of the McKay-Thompson series in the context of Monstrous moonshine [40] (see equ. (B.4)). Indeed, for any element $g \in \mathbb{M}_{24}$ we can define ${ }^{4}$

$$
\phi_{g}(\tau, z):=\frac{1}{2} \operatorname{Tr}_{R R}\left((-1)^{F+\bar{F}} g y^{J_{0}} q^{L_{0}-\frac{c}{24}} \bar{q}^{\bar{L}_{0}-\frac{c}{24}}\right) .
$$

Although $\mathbb{M}_{24}$ has of order $10^{8}$ elements, they fall into only 26 conjugacy classes (see table 1 for the full character table), such that there are a priori only 26 different twining genera. ${ }^{5}$ If the conjectured expression (2.9) is indeed correct, it should be possible to write $\phi_{g}$ in the following manner

$$
\begin{gathered}
\phi_{g}(\tau, z):=\frac{1}{2}\left[\operatorname{Tr}_{H_{00}}(g) \operatorname{ch}_{h=\frac{1}{4}, l=0}^{\mathscr{N}=4}(\tau, z)-\operatorname{Tr}_{H_{0}}(g) \operatorname{ch}_{h=\frac{1}{4}, l=\frac{1}{2}}^{\mathscr{N}=4}(\tau, z)\right. \\
\left.+\sum_{n=1}^{\infty} \operatorname{Tr}_{H_{n}}(g) \operatorname{ch}_{h=n+\frac{1}{4}, l=\frac{1}{2}}^{\mathscr{N}=4}(\tau, z)\right]
\end{gathered}
$$

with the first few representations $H_{n}$ as given in (2.10). Similar to the elliptic genus itself, it was first argued in $[20,21]$ using standard conformal field theory methods, that the twining genera $\phi_{g}$ should transform as weak Jacobi forms of index 1 and weight 0 under some congruence subgroup of $S L(2, \mathbb{Z})$, up to some multiplier system. Indeed, in [22] it was conjectured that for any $g \in \mathbb{M}_{24}$ of order $N$

$$
\phi_{g}\left(\frac{a \tau+b}{c \tau+d}, \frac{z}{c \tau+d}\right)=e^{\frac{2 \pi i c d}{N h}} e^{\frac{2 \pi i c z^{2}}{c \tau+d}} \phi_{g}(\tau, z), \quad \text { for } \quad\left(\begin{array}{c}
a b \\
c d
\end{array}\right) \in \Gamma_{0}(N)
$$

where $\Gamma_{0}(N)$ is the congruence subgroup of $S L(2, \mathbb{Z})$ defined as

$$
\Gamma_{0}(N):=\left\{\left(\begin{array}{l}
a b \\
c d
\end{array}\right) \in S L(2, \mathbb{Z}) \mid c \equiv 0 \bmod N\right\}
$$

\footnotetext{
${ }^{4}$ For convenience we have included a factor $\frac{1}{2}$, such that $\phi_{1 A}(\tau, z)=\frac{1}{2} \phi_{K 3}(\tau, z)=\phi_{0,1}(\tau, z)$ is identical to the standard weak Jacobi form of index 1 and weight 0 (see [35]).

${ }^{5}$ Due to the fact that there are 10 conjugacy classes which appear in complex conjugate pairs, there are only 21 distinct twining genera.
} 
The quantity $h \in \mathbb{N}$ with $h \mid \operatorname{gcd}(N, 12)$ determines the multiplier system, which is in particular trivial if $g$ contains a representative of $\mathbb{M}_{23} \subset \mathbb{M}_{24}$. I.e. $h \neq 1$ only for those conjugacy classes which reside to the right of conjugacy class $23 \mathrm{~B}$ in the character table given in table 1 .

Besides the conjectured transformation properties (2.12), we also know the first few Fourier coefficients for each twining genus: They can be inferred from the first few representations in equation (2.10) that follow from the original observation of [19]. It turns out that these two pieces of information are sufficient to obtain closed form expressions for all $\phi_{g}$. A number of them have first been worked out in [20,21] while the complete list has been given in [22, 23].

The information encoded in the $\phi_{g}(\tau, z)$ in turn can be used to find explicit decompositions of the representations $H_{n}$ (for $n>6$ ) into irreducible representations of $\mathbb{M}_{24}$. It has been checked up to $n=600$ (see $[22,23]$ ) that the multiplicities in this decomposition are indeed non-negative integer coefficients as is required for well defined representations. This highly non-trivial result provides overwhelming evidence for the correctness of the Mathieu-moonshine conjecture, albeit it is no full-fledged mathematical proof.

\section{Symmetries of $K 3$ Sigma Models}

\subsection{Quantum Symmetries}

Given the strong evidence in favour of the conjecture (2.8) an important question is why exactly $\mathbb{M}_{24}$ seems to be acting on the BPS states contributing to the elliptic genus of $K 3$. An important theorem in this respect was derived in [41]

Theorem: Let $G$ be a finite group of symplectic automorphisms of a K3 surface. Then $G$ is isomorphic to a subgroup of the Mathieu group $\mathbb{M}_{23}$, which has at least 5 orbits on a set of 24 elements.

This suggests that an action of $\mathbb{M}_{23} \subset \mathbb{M}_{24}$ on the space of BPS states contributing to the elliptic genus may be expected from a geometric point of view. However, in the expansion (2.9) we encounter irreducible representations of $\mathbb{M}_{24}$ rather than merely $\mathbb{M}_{23}$. It therefore seems that the purely geometric action gets enhanced at the quantum level. A natural first question is therefore how symmetries of sigma models with $K 3$ target space are related to the Mathieu group $\mathbb{M}_{24}$.

Recalling the conjectured structure of $\mathscr{H}^{\text {BPS }}$ from equ. (2.8), however, we are not interested in any possible symmetry of the sigma model, but only in those which commute with the $\mathscr{N}=(4,4)$ superconformal algebra. To classify these symmetries let us first consider the moduli space of $\mathscr{N}=(4,4)$ theories with elliptic genus $\phi_{K 3}$, which is believed to be $[42,43]$

$$
\mathscr{M}_{K 3}=O\left(\Gamma^{4,20}\right) \backslash O(4,20) /(O(4) \times O(20)) .
$$

The Grassmannian $O(4,20) /(O(4) \times O(20))$ is parametrized by the choice of a positive definite 4-dimensional subspace $\Pi \subset \mathbb{R}^{4,20}$ and $O\left(\Gamma^{4,20}\right)$ is the group of automorphisms of the even unimodular lattice $\Gamma^{4,20} \subset \mathbb{R}^{4,20}$. Physically, we may think of $\Gamma^{4,20}$ as the integral homology of $K 3$, i.e. the lattice of D-brane charges, and the position of $\Pi$ is fixed through the choice of a Ricci-flat metric and a $B$-field on $K 3$. As was discussed in [24] the group of supersymmetry preserving automorphisms $G \equiv G_{\Pi}$ of the non-linear sigma-model characterized by $\Pi$ consists of those elements of 
$O\left(\Gamma^{4,20}\right)$ which leave $\Pi$ invariant pointwise. Let us introduce the following orthogonal sublattices in $\Gamma^{4,20}$

$$
\begin{aligned}
& L^{G}:=\left\{x \in \Gamma^{4,20} \mid g(x)=x, \forall g \in G\right\}, \\
& L_{G}:=\left\{x \in \Gamma^{4,20} \mid x \cdot y=0, \forall y \in L^{G}\right\} .
\end{aligned}
$$

By construction, $\Pi$ is a subspace of the real vector space $L^{G} \otimes \mathbb{R} \subset \mathbb{R}^{4,20}$, and since $\Pi$ has signature $(4,0)$, the orthogonal complement $L_{G}$ must be a negative definite lattice of rank at most 20 . Therefore, as was explained in [24], $L_{G}(-1)$ can be embedded into the Leech lattice $\Lambda$, provided that $L_{G}(-1)$ does not contain any vectors of length squared two. ${ }^{6}$ Since the action of $G$ fixes all vectors of $\Lambda$ orthogonal to $L_{G}(-1), G$ must be a subgroup of $C o_{1} \subset C o_{0}=\operatorname{Aut}(\Lambda)$ that fixes pointwise a sublattice of the Leech lattice of rank at least 4 . A more careful analysis then leads to the theorem [24]

Theorem: Let $G$ be the group of symmetries of a non-linear $\sigma$-model on $K 3$ preserving the $\mathscr{N}=(4,4)$ superconformal algebra. Then one of the following possibilities holds:

1. $G=G^{\prime} \cdot G^{\prime \prime}$, where $G^{\prime} \subset \mathbb{Z}_{2}^{11}$, and $G^{\prime \prime} \subset \mathbb{M}_{24}$ with at least four orbits when acting as a permutation on $\{1, \ldots, 24\}$

2. $G=5^{1+2} \cdot \mathbb{Z}_{4}$

3. $G=\mathbb{Z}_{3}^{4} \cdot A_{6}$

4. $G=3^{1+4} \cdot \mathbb{Z}_{2} \cdot G^{\prime \prime}$, where $G^{\prime \prime}$ is either trivial, $\mathbb{Z}_{2}, \mathbb{Z}_{2}^{2}$ or $\mathbb{Z}_{4}$.

Here $p^{1+2 n}$ denotes an extra special group of order $p^{1+2 n}$, and $N . Q$ denotes a group $G$ for which $N$ is a normal subgroup such that $G / N \cong Q$. Except for case 1 . with $G^{\prime}$ trivial, these groups are not subgroups of $\mathbb{M}_{24}$.

This classification does not uniquely single out $\mathbb{M}_{24}$ but includes several other options (particularly cases 2.-4.). In fact, in [24] a particular Gepner model has been studied in which possibility 3. is indeed realized. This result is somewhat puzzling, even more so since all cases of the classification theorem are subgroups of the Conway group $\mathrm{Co}_{1}$, which, however, seems not to be favored by the elliptic genus of $K 3$. Therefore, the appearance of $\mathbb{M}_{24}$ in the space of BPS states of $K 3$ sigma-models must be more involved and further insights seem to be necessary. For example, we might hope that we can gain further hints by studying the role of $\mathbb{M}_{24}$ from the target space point of view.

\section{1/4-BPS Saturated String Amplitudes}

\subsection{Connection to Target Space Physics and BPS States in String Theory}

So far our attempts to understand the appearance of $\mathbb{M}_{24}$ have focused on the world-sheet (i.e. conformal field theory) side. It is a natural question to see, whether we may also gather insights by using a space-time (effective action) approach. Indeed, we would like to understand how much

\footnotetext{
${ }^{6}$ The presence of the latter would signal an enhancement of gauge symmetry and would lead to a singular CFT [42].
} 
(if any) of the action of $\mathbb{M}_{24}$ maps over to the target space physics. To this end, we first need to identify a suitable quantity on the target space which might display $\mathbb{M}_{24}$ symmetry, i.e. some BPS saturated object that is related to the elliptic genus of $K 3$.

In the following we will discuss such a quantity, namely a particular BPS saturated amplitude in type II string theory compactified on $K 3 \times T^{2}$, whose integrand can be written as derivatives of $\phi_{K 3}$. However, before discussing the amplitude explicitly, let us first review the BPS spectrum of this particular string compactification. The effective action is described by $\mathscr{N}=4$ supergravity in four dimensions. The massless spectrum comprises the $\mathscr{N}=4$ supergravity multiplet coupled to 28 vector multiplets. The latter consist of a vector field (gaugino), four Weyl spinors and six real scalars. However, not all of the $28 \mathrm{VMs}$ are physical. Indeed, 6 of them act as compensating multiplets: As explained in e.g. [2], the 36 scalars of these multiplets are eliminated by imposing the D-term constraints (20 constraints) as well as gauge fixing Weyl invariance (one constraint) and local $\mathrm{SO}(6)$ symmetry (15 constraints). The remaining 134 physical scalars ${ }^{7}$ from the Weyl multiplet and the remaining $22 \mathrm{VMs}$ span the coset space:

$$
\frac{S U(1,1)}{U(1)} \otimes \frac{S O(22,6, \mathbb{R})}{S O(22, \mathbb{R}) \times S O(6, \mathbb{R})} .
$$

The first factor corresponds to the $T^{2}$ torus and is described by the Kähler modulus $T=T_{1}+$ $i T_{2}$, while its complex structure modulus $U=U_{1}+i U_{2}$, the $\sigma$-model moduli of $K 3$, the type IIA dilaton $S$ and the Wilson lines on $T^{2}$ of the Ramond-Ramond gauge fields parametrize the second factor. Since in the type II compactification two supercharges from the left- and right-movers each comprise the full $\mathscr{N}=4$ SUSY algebra, half of the gauginos originate from the R-NS sector and the second half from the NS-R sector.

As discussed in [44] (see also [16] for a more explicit exhibition of these states), in type IIA the 22 physical gauge vectors in the VMs arise from the R-R 3-form potentials reduced on the $b_{2}(K 3)=22$ two-cycles, while the six graviphotons from the supergravity multiplet stem from the R-R 1-form in 10 dimensions, the R-R 3-form reduced on $T^{2}$, the NS-NS anti-symmetric tensor and metric reduced along the two one-cycles of the torus $T^{2}$. We can associate electric and magnetic charges to all these gauge fields. In particular, the fundamental string wrapped on $T^{2}$ with winding numbers $n_{1}, n_{2}$ and Kaluza-Klein (KK) momenta $m_{1}, m_{2}$ is electrically charged under the reduction of the 10-dimensional B-field and the metric on $T^{2}$ with charges $n_{1}, n_{2}, m_{1}, m_{2} \in \mathbb{Z}$, respectively. ${ }^{8}$ The mass of a fundamental string state is given by

$$
m_{L}^{2}=N_{L}-\frac{1}{2}+\left|P_{L}\right|^{2}+\left(p_{\mu}\right)^{2}, \quad \text { and } \quad m_{R}^{2}=N_{R}-\frac{1}{2}+\left|P_{R}\right|^{2}+\left(p_{\mu}\right)^{2},
$$

with $p_{\mu}$ the space-time momentum, $N_{L, R}$ the left- and right-moving oscillator numbers and the Narain momenta $\left(P_{L}, P_{R}\right) \in \Gamma^{2,2}$

$$
P_{L}=\frac{1}{\sqrt{2 T_{2} U_{2}}}\left(m_{1}+m_{2} U+n_{1} \bar{T}+n_{2} \bar{T} U\right), \quad \text { and } \quad P_{R}=\frac{1}{\sqrt{2 T_{2} U_{2}}}\left(m_{1}+m_{2} U+n_{1} T+n_{2} T U\right) .
$$

\footnotetext{
${ }^{7}$ Physically, in type IIA these scalars arise as follows: The $\sigma$-model of $K 3$ has 80 and that of $T^{2}$ four real deformations. The R-R 1-form gives rise to the two real scalars $C_{4}, C_{5}$ and the R-R 3-form gives $b_{3}\left(K 3 \times T^{2}\right)=44$ scalars. Reducing the R-R 3-form down to an anti-symmetric space-time 2-tensor, which can be dualized to a scalar, gives $b_{1}\left(K 3 \times T^{2}\right)=2$ more scalars. Together with the dilaton field $S$ we obtain $80+4+2+44+2+2=134$ real scalars.

${ }^{8}$ Their corresponding magnetic counter parts are described by the NS five-brane wrapped on $K_{3} \times S^{1}$ and a KK monopole on $S^{1}$.
} 
Level matching requires the identifications

$$
m_{L}^{2}-m_{R}^{2}=N_{L}-N_{R}+\left|P_{L}\right|^{2}-\left|P_{R}\right|^{2}=N_{L}-N_{R}+2\left(m_{1} n_{2}-n_{1} m_{2}\right)=0 .
$$

The class of states which will be relevant for us in the following are the so-called DabholkarHarvey (DH) states. The latter are perturbative BPS states, which may either have left-moving $N_{L}$ or right-moving $N_{R}$ excitations [11]:

$$
\begin{array}{rccc}
N_{R}, N_{L}=0: & m_{1} n_{2}-n_{1} m_{2}=0, & 1 / 2 \mathrm{BPS}, \\
N_{L}=0: & 2\left(m_{1} n_{2}-n_{1} m_{2}\right)=N_{R}, & 1 / 4 \mathrm{BPS}, \\
N_{R}=0: & 2\left(n_{1} m_{2}-m_{1} n_{2}\right)=N_{L}, & 1 / 4 \mathrm{BPS} .
\end{array}
$$

Depending on the value of the duality invariant $m_{1} n_{2}-n_{1} m_{2}$ these states represent either $1 / 2$ or $1 / 4$ BPS saturated string states. In [44] particular string theory amplitudes have been discussed which only receive contributions from such states and which are intimately related to the elliptic genus of $K 3$. Such amplitudes have to be very carefully arranged, to furnish all the properties that we have reviewed in section 2.1. First of all, since $\phi_{K 3}$ is a weak Jacobi form of $S L(2, \mathbb{Z})$ the amplitude we are interested in must appear at one-loop in string perturbation theory. ${ }^{9}$ Moreover, since $\phi_{K 3}$ is a holomorphic function in both arguments, the amplitude cannot be left-right symmetric. Indeed, as reviewed in section 2.1, it can only depend on the right moving ground state, which means that only zero modes may contribute from the right moving sector. The left-moving sector, however, is free to receive any type of BPS excitations, such that in fact we have two different possibilities

- all external states are $1 / 2$ BPS

- we may also allow for $1 / 4$ BPS external states

As we shall see in the explicit computations, in the first case, the integrand will only be sensitive to the contributions of short multiplets to the elliptic genus, while in the second case, we will encounter the full $\phi_{K 3}$.

\subsection{1/2 BPS Saturated Amplitude}

\subsubsection{General Setup}

In [44] the following class of 1/2 BPS saturated one-loop couplings has been proposed

$$
\mathscr{G}_{N} R_{(+), \mu v \rho \tau \sigma} R_{(+)}^{\mu v \rho \sigma}\left(F_{(-), \lambda \tau} F_{(-)}^{\lambda \tau}\right)^{N}, \quad N \in \mathbb{N}
$$

with $R_{(+)}^{\mu v \rho \sigma}$ the self-dual part of the four-dimensional Riemann tensor and $F_{(-)}^{\mu v}$ the field strength of the Kaluza-Klein vector field coming from the compactification on $T^{2}$ and $N$ is an arbitrary integer. The coupling function $\mathscr{G}_{N}$ can be found by a one-loop computation with $2 N+2$ external

\footnotetext{
${ }^{9}$ Here we will consider $\phi_{K 3}$ as a function of the world-sheet torus coordinate $\tau$ and a further variable $z$ which we will encounter later on.
} 
states. The explicit answer found in [44] is given as

$$
\begin{gathered}
\mathscr{G}_{N}=\left.4 \int \frac{d^{2} \tau}{\tau_{2}} \tau_{2}^{2 N} \sum_{n=0}^{N}\left(\begin{array}{l}
2 N \\
2 n
\end{array}\right) \frac{\partial^{2 n}}{\partial z^{2 n}} \frac{\partial^{2 N-2 n}}{\partial w^{2 N-2 n}}\left[\left(\frac{2 \pi i w \eta(\tau)^{3}}{\theta_{1}(w, \tau)}\right)^{2} \phi_{K 3}(\tau, z) e^{-\frac{\pi\left(w^{2}+z^{2}\right)}{\tau_{2}}}\right]\right|_{z=w=0} \\
\times \sum_{\Gamma^{(2,2)}}\left(P_{R}\right)^{2 N} q^{\frac{1}{2}\left|P_{L}\right|^{2}} \bar{q}^{\frac{1}{2}\left|P_{R}\right|^{2}}
\end{gathered}
$$

By a change of variables, $\mathscr{G}_{N}$ can also be written as

$$
\mathscr{G}_{N}=-\left.16 \pi^{2} \int \frac{d^{2} \tau}{\tau_{2}} \tau_{2}^{2 N} \frac{\partial^{2 N}}{\partial u^{2 N}}\left[\frac{u^{2} \eta(\tau)^{6}}{\theta_{1}(u, \tau)^{2}} \phi_{K 3}(\tau, u) e^{-\frac{2 \pi u^{2}}{\tau_{2}}}\right]\right|_{u=0} \sum_{\Gamma^{(2,2)}}\left(P_{R}\right)^{2 N} q^{\frac{1}{2}\left|P_{L}\right|^{2}} \bar{q}^{\frac{1}{2}\left|P_{R}\right|^{2}} .
$$

As we can see, the integrand of (4.7) is indeed related to the elliptic genus of $K 3$. As has been argued in [44], it is in fact just the contribution of the short multiplets in the expansion (2.5) with $\ell=0$. To see this, we drop the factor $e^{-\frac{2 \pi u^{2}}{\tau_{2}}}$, which corresponds to a non-analytic contribution to the integral (see [44] for a detailed discussion on this point). Moreover we use the explicit form of the $\mathscr{N}=4$ characters (2.6) and (2.7) to write

$$
\phi_{K 3}(\tau, u)=\frac{\theta_{1}(\tau, u)^{2}}{\eta(\tau)^{3}}[24 \mu(\tau, u)+\Sigma(\tau)],
$$

where we have introduced

$$
\Sigma(\tau)=-8\left[\mu\left(\tau, u=\frac{1}{2}\right)+\mu\left(\tau, u=\frac{1+\tau}{2}\right)+\mu\left(\tau, u=\frac{\tau}{2}\right)\right]=-2 q^{-\frac{1}{8}}\left(1-\sum_{n=1}^{\infty} A_{n} q^{n}\right) .
$$

We notice in particular that, since $\Sigma(\tau)$ is independent of $z, \mathscr{G}_{N \geq 1}$ will only receive contributions from the first term in the square bracket of (4.8). The latter, however, is precisely the contribution of the massless $\mathscr{N}=4$ multiplets to the elliptic genus.

\section{Intermediate Multiplets and the Elliptic Genus}

A natural question to ask is whether it is possible to slightly modify the coupling (4.5) in such a manner as to capture the full elliptic genus of $K 3$, namely also the $1 / 4$ BPS contributions. To this end, we recall that (4.5) only involves external states from short (i.e. 1/2 BPS) multiplets and we therefore may expect qualitatively different results when we allow external states from intermediate or long multiplets. This can be achieved in two different ways: (i) through so-called reducible diagrams, i.e. amplitudes with two of the external vertices colliding (see figure 2 for an example) and intermediate states propagating in the internal channels, or (ii) directly using massive external states. In [44] it has been shown, that both approaches effectively lead to the same result and we will therefore only focus on approach (ii).

Indeed, the type of amplitude proposed in [44] is of the following form

$$
\mathscr{F}_{N}=\left\langle\int d^{2} z_{1} V_{R}^{(0,0)}\left(h_{11}, p_{2}\right) \int d^{2} z_{2} V_{R}^{(0,0)}\left(h_{\overline{1}}, \bar{p}_{2}\right) \prod_{a=1}^{N} \int d^{2} x_{a} V_{M}^{(-1,-1)}\left(p_{a}, x_{a}, \bar{x}_{a}\right) \prod_{b=1}^{N} V_{\mathrm{PCO}}\right\rangle
$$




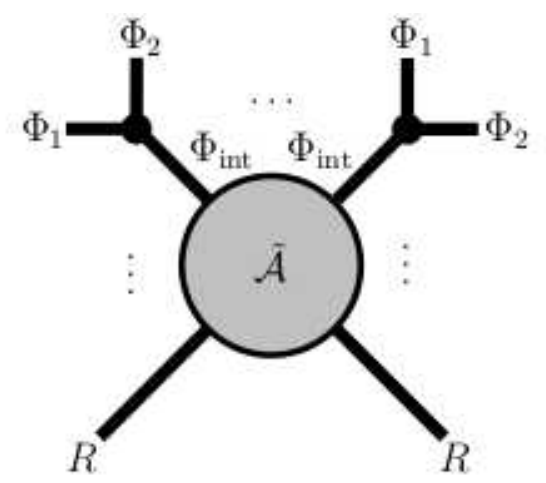

where

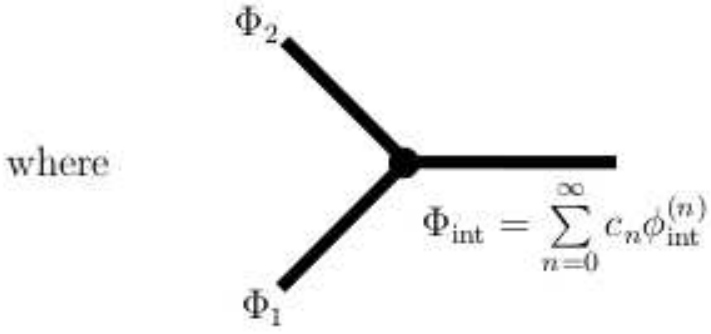

Figure 2: Reducible diagram with external fields $\Phi_{1}$ and $\Phi_{2}$ pairwise colliding. The intermediate channel can be decomposed into a series of fields of different conformal dimensions, belonging to different multiplets.

where $V_{R}^{(0,0)}\left(h_{11}, p_{2}\right)$ are the vertex operators of gravitons with polarization $h_{\mu v}$ and momentum $p_{\mu}$, while $V_{M}^{(-1,-1)}$ are vertex operators for particular massive scalar fields, whose precise form is given by ${ }^{10}$

$$
V_{M}^{(-1,-1)}(p, z, \bar{z})=: e^{-\varphi} \psi_{3} \partial H(z) e^{-\tilde{\varphi}} \tilde{\psi}_{3} \bar{\partial} X_{3}(\bar{z}) e^{i p X}:
$$

We refer the reader to [44] for further technical details on these massive vertex operators but just remark that $\psi_{3}$ and $X_{3}$ are free fermions and bosons of the world-sheet theory of $T^{2}$, while $\varphi$ bosonizes the superghost and $H$ the $U(1) \subset S U(2)$ current of the internal $K 3$ world-sheet theory. The state corresponding to (5.2) is a massive modulus field from the $T^{2}$ compactification, however, from the point of view of the internal CFT living on $K 3$, it is in the right-moving ground state. This in fact is the reason why the string amplitudes will finally only be sensitive to the very basic topological information of K3, i.e. the elliptic genus. Explicit computation in [44] leads to the following result

$$
\mathscr{F}_{N}=\int \frac{d^{2} \tau}{\tau_{2}} \tau_{2}^{2 N}\left[\frac{\partial^{N}}{\partial z^{N}} \phi_{K 3}(\tau, z)\right]_{z=0} \sum_{\left(P_{L}, P_{R}\right) \in \Gamma^{2,2}}\left(P_{L}\right)^{N}\left(P_{R}\right)^{2 N} q^{\frac{1}{2}\left|P_{L}\right|^{2}} \bar{q}^{\frac{1}{2}\left|P_{R}\right|^{2}}
$$

As it stands, this expression is not very illuminating since it still involves the integration over $\tau$. To perform the latter we first realize that one may write

$$
\mathscr{F}_{N}(T, U)=\left(\mathscr{D}_{\bar{U}}\right)^{N} \int \frac{d^{2} \tau}{\tau_{2}}\left[\frac{\partial^{N}}{\partial z^{N}} \phi_{K 3}(\tau, z)\right]_{z=0} \sum_{\substack{m_{1}, m_{2} \in \mathbb{Z} \\ n_{1}, n_{2} \in \mathbb{Z}}}^{\prime}\left(\tau_{2} P_{R}\right)^{N} q^{\frac{1}{2}\left|P_{L}\right|^{2}} \bar{q}^{\frac{1}{2}\left|P_{R}\right|^{2}}=\left(\mathscr{D}_{\bar{U}}\right)^{N} \tilde{\mathscr{F}}_{N}(T, U),
$$

where we have introduced the covariant differential $\mathscr{D}_{\bar{U}}$ with respect to the modulus $U$ which acts in the following manner on a function $f^{(w)}$ of weight $w$

$$
\mathscr{D}_{\bar{U}} f^{(w)}=\frac{U-\bar{U}}{2}\left(\frac{\partial}{\partial \bar{U}}-\frac{w}{2(U-\bar{U})}\right) f^{(w)} .
$$

\footnotetext{
${ }^{10}$ In $(5.1)$ these vertices appear in the $(-1)$ ghost picture, while the gravitons are inserted in the (0)-picture. To balance the total ghost number, we also inserted $N$ picture changing operators $V_{\mathrm{PCO}}(r, \bar{r})$.
} 
Here the prefactor takes into account the normalization of the $U$-vertex operators in string theory (see e.g. [11]) and the second term in the bracket yields the curvature contribution. With this we can now define the following generating functional (with the expansion parameter $\lambda$ )

$$
\mathbb{F}(\lambda, T, U)=\sum_{N=0}^{\infty} \frac{\left(\frac{\lambda}{\sqrt{2 T_{2} U_{2}}}\right)^{N}}{N !} \tilde{\mathscr{F}}_{N}(T, U)=\int \frac{d^{2} \tau}{\tau_{2}} \sum_{\substack{m_{1}, m_{2} \in \mathbb{Z} \\ n_{1}, n_{2} \in \mathbb{Z}}}^{\prime} \phi_{K 3}\left(\tau, \frac{\lambda \tau_{2} P_{R}}{\sqrt{2 T_{2} U_{2}}}\right) q^{\frac{1}{2}\left|P_{L}\right|^{2}} \bar{q}^{\frac{1}{2}\left|P_{R}\right|^{2}}
$$

Upon Fourier expanding the elliptic genus of $K 3$

$$
\phi_{K 3}(\tau, z)=\sum_{n, \ell} c(n, \ell) q^{n} e^{2 \pi i z \ell}, \quad \text { with } \quad c(n, \ell)=c\left(n-\frac{\ell^{2}}{4}\right)
$$

the $\tau$-integral in the generating functional (5.5) can be performed explicitly ${ }^{11}$, yielding [44]

$$
\mathbb{F}(\lambda, T, U)=-4 \ln \left[(\kappa Y)^{10}\left|e^{2 \pi i(T+U+i \lambda)} \prod_{r, n^{\prime}, \ell>0}\left(1-e^{2 \pi i\left(r T+n^{\prime} U+i \ell \lambda\right)}\right)^{c\left(n^{\prime} r, \ell\right)}\right|\right],
$$

where $Y=T_{2} U_{2}-\lambda^{2}$. Comparing this expression with [48], we recognize that

$$
\chi_{10}(T, U ; \lambda)=e^{2 \pi i(T+U+i \lambda)} \prod_{r, n^{\prime}, \ell>0}\left(1-e^{2 \pi i\left(r T+n^{\prime} U+i \ell \lambda\right)}\right)^{c\left(n^{\prime} r, \ell\right)}
$$

is nothing but the product representation of the unique weight 10 Igusa cusp form ${ }^{12}$ of $S O(2,3 ; \mathbb{Z}) \simeq$ $S p(4, \mathbb{Z})$. We also note that the remaining terms in (5.7) guarantee covariance of the integral under this latter group. Finally, the group $S O(2,3, \mathbb{Z})$ is parametrized by the torus moduli $(T, U)$ (which generically give rise to $S O(2,2, \mathbb{Z}))$ and the additional coupling constant $\lambda$.

\section{Conclusions}

In this article we have reviewed recent interesting studies of the elliptic genus of $K 3$ as an example of a BPS saturated quantity in string theory. We have used a world-sheet as well as a target-space approach to study the space of BPS states contributing to the elliptic genus of $K 3$.

In the CFT approach we have discussed some recently discovered algebraic properties, which go by the name of Mathieu moonshine, acknowledging similar preceding discoveries in connection with the Monster sporadic group. Although there is so far no abstract proof for Mathieu moonshine we have reviewed the overwhelming evidence which has recently been compiled in its favour. In order to understand the action of the Mathieu group $\mathbb{M}_{24}$ on the elliptic genus of $K 3$ it is necessary to obtain a better understanding of sigma-models with $K 3$ target space at the quantum level. We have reviewed a classification theorem derived in [24] for all discrete symmetries which leave the $\mathscr{N}=(4,4)$ supersymmetry intact. Unfortunately, this classification does not uniquely single out $\mathbb{M}_{24}$ but includes several other options as well. In fact, in [24] a particular Gepner model has been

\footnotetext{
${ }^{11}$ To solve this integral, in [44] the method of orbits was used, which was first introduced in [45, 13] and further developed in $[46,11,47]$.

${ }^{12}$ This genus two modular form first appeared in string theory to describe the bosonic two-loop partition function [49] and in the context of one-loop gauge threshold corrections [50].
} 
studied in which one of the latter is indeed realized. This result is somewhat puzzling and probably indicates that the appearance of $\mathbb{M}_{24}$ is slightly more involved.

From the target space point of view we have presented several BPS saturated amplitudes whose integrands are linked to the elliptic genus of $K 3$. We have seen two different types of amplitudes: those with external fields from massless short multiplets only capture a particular part of the elliptic genus corresponding to a mock modular form. As was argued in [44] (see also [36]) this part is linked to the elliptic genus of massless short multiplets. The full elliptic genus is obtained from amplitudes with external fields sitting in massive multiplets. It would be interesting to understand whether the appearance of the elliptic genus in string amplitudes signals an action of $\mathbb{M}_{24}$ on the BPS states contributing to these couplings (see [25, 29] for related discussions). Moreover, in [44] a generating functional for these later amplitudes has been worked out, which is the weight 10 cusp form of $S p(4, \mathbb{Z})$. In Ref. [51] $\chi_{10}$ has been identified as the partition function counting dyons (dyonic five-brane states) in $\mathscr{N}=4$ string theory and it was argued that $\chi_{10}$ can be related to the free energy of strings in $D=6$ with target-space $K 3 \times T^{2}$. It would be interesting to find a possible connection between the one-loop amplitude of [44] and the computation of this free energy from the partition function of the six-dimensional world-volume string theory on $K 3 \times T^{2}$.

Besides this it would also be nice to see, whether the twining genera defined in (2.11) also appear in string theory scattering amplitudes. This would probably give a more direct hint on the appearance of $\mathbb{M}_{24}$ in string theory. Finally, it would be very curious to see whether it is also possible to define a generating functional for twining genera, similar to the Igusa cusp-form $\chi_{10}$ for the elliptic genus. It would be very interesting to see whether any connection can be made to the modular forms recently discussed in [52].

\section{Acknowledgements}

I would like to thank the organizers of the Corfu Summer Institute 2011 for giving me the opportunity to present this work and for creating a highly stimulating atmosphere throughout the whole workshop.

\section{A. The Character Table of the Mathieu Group $\mathbb{M}_{24}$}

$\mathbb{M}_{24}$ is th largest of the Mathieu groups and can best be thought of as a subgroup of $S_{24}$ the permutation group of 24 elements. It is a sporadic (finite and simple) group with

$$
2^{10} \cdot 3^{3} \cdot 5 \cdot 7 \cdot 11 \cdot 23=244823040
$$

elements. The latter are organized in 26 different conjugacy classes, which we will denote using standard ATLAS notation, i.e. by a number, which indicates the order of the group element, and a capital letter. The full character table can be found in Table 1 . 


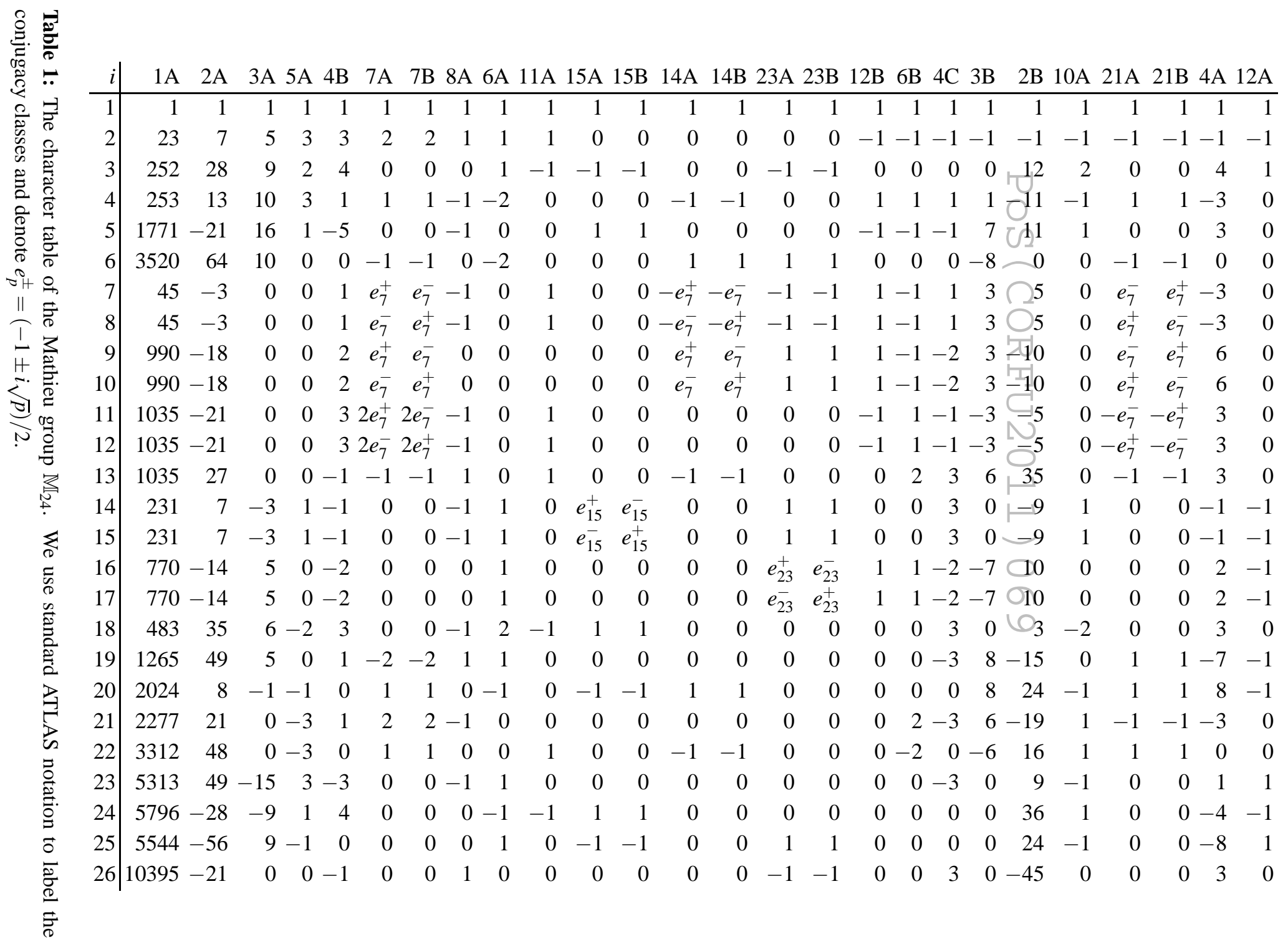




\section{B. Monstrous Moonshine}

Consider the Fourier expansion of the modular invariant $J$-function

$$
\begin{aligned}
J(\tau): & =\frac{E_{4}(\tau)^{3}}{\eta(\tau)^{24}}-744 \\
& =\frac{1}{q}+196884 q+21493760 q^{2}+864299970 q^{3}+\mathscr{O}\left(q^{4}\right),
\end{aligned}
$$

where $E_{4}(\tau)$ is the Eisenstein series of weight 4 and $\eta(\tau)$ the Dedekind-eta function. In 1978 McKay and Thompson observed that the coefficients can be decomposed in the following manner

$$
\begin{aligned}
196884 & =196883+1, \\
21493760 & =21296876+196883+1, \\
864299970 & =842609326+21296876+2 \cdot 196883+2 \cdot 1,
\end{aligned}
$$

where the numbers that appear on the right are the dimensions of the smallest irreducible representations of the Fischer-Griess Monster group $\mathfrak{M}$. This observation gives an intriguing hint to a connection between modular forms and sporadic groups and suggests the existence of an infinitedimensional graded representation

$$
V=V_{-1} \oplus V_{1} \oplus V_{2} \oplus V_{3} \oplus \ldots
$$

with $V_{n}$ representations of $\mathfrak{M}$ such that

$$
J(\tau)=\operatorname{dim}\left(V_{-1}\right) q^{-1}+\sum_{n=1}^{\infty} \operatorname{dim}\left(V_{n}\right) q^{n} .
$$

In [40] a more generalized object than $J(\tau)$ was proposed in which the dimensions of $V_{n}$ are replaced by characters $\operatorname{ch}_{V_{n}}(g)=\operatorname{Tr}_{V_{n}}(g)$ for elements $g \in \mathfrak{M}$

$$
T_{g}(\tau):=\operatorname{ch}_{V_{-1}}(g) q^{-1}+\sum_{n=1}^{\infty} \operatorname{ch}_{V_{n}}(g) q^{n} .
$$

There are 171 distinct such McKay-Thompson series which have been shown to be Hauptmoduls of various genus 0 subgroups $\Gamma$ of $S L(2, \mathbb{Z}) .^{13}$

There is an elegant explanation for these observations[53, 46, 54]: $J(\tau)$ can be interpreted as the partition function of a (bosonic) conformal field theory with central charge $c=24$, whose automorphism group is the Monster group. Indeed, the space of states of this CFT takes the form

$$
V^{\natural}=\bigoplus_{n=0}^{\infty}\left(V_{n} \otimes \mathscr{H}_{h=n}^{\mathrm{Vir}}\right),
$$

where $V_{n}$ are representations of the Monster group, while $\mathscr{H}_{h=n}^{\mathrm{Vir}}$ are representations of the Virasoro algebra with conformal weight $h$.

\footnotetext{
${ }^{13}$ Genus zero in this context means that $\mathbb{H}_{+} / \Gamma$ has the topology of a sphere. The Hauptmodul is the corresponding function which encodes the change of coordinates to the Riemann sphere $\mathbb{C} \cup\{\infty\}$ (see [39] for a more detailed discussion).
} 


\section{References}

[1] I. Antoniadis, E. Gava, K. S. Narain and T. R. Taylor, Topological amplitudes in string theory, Nucl. Phys. B 413 (1994) 162 [hep-th/9307158].

[2] I. Antoniadis, S. Hohenegger, K. S. Narain and E. Sokatchev, Harmonicity in N=4 supersymmetry and its quantum anomaly, Nucl. Phys. B 794 (2008) 348 [arXiv:0708.0482 [hep-th]].

[3] M. Bershadsky, S. Cecotti, H. Ooguri and C. Vafa, Kodaira-Spencer theory of gravity and exact results for quantum string amplitudes, Commun. Math. Phys. 165 (1994) 311 [hepth/9309140].

[4] T. W. Grimm, A. Klemm, M. Marino and M. Weiss, Direct Integration of the Topological String, JHEP 0708 (2007) 058 [hep-th/0702187];

M.x. Huang and A. Klemm, Holomorphic anomaly in gauge theories and matrix models, JHEP 0709 (2007) 054 [arXiv:hep-th/0605195];

M.x. Huang, A. Klemm and S. Quackenbush, Topological String Theory on Compact CalabiYau: Modularity and Boundary Conditions, Lect. Notes Phys. 757 (2009) 45 [arXiv:hepth/0612125].

[5] I. Antoniadis, E. Gava, K.S. Narain and T.R. Taylor, Topological amplitudes in heterotic superstring theory, Nucl. Phys. B476 (1996) 133 [hep-th/9604077].

[6] J.F. Morales and M. Serone, Higher derivative F terms in N=2 strings, Nucl. Phys. B481 (1996) 389 [hep-th/9607193];

I. Antoniadis, S. Hohenegger, K.S. Narain and T.R. Taylor, Deformed Topological Partition Function and Nekrasov Backgrounds, Nucl. Phys. B838 (2010) 253 [arXiv:1003.2832 [hepth]].

[7] I. Antoniadis, S. Hohenegger, K.S. Narain and E. Sokatchev, A New Class of N=2 Topological Amplitudes, Nucl. Phys. B 823 (2009) 448 [arXiv:0905.3629 [hep-th]].

[8] I. Antoniadis, S. Hohenegger, K. S. Narain and E. Sokatchev, Generalized N=2 Topological Amplitudes and Holomorphic Anomaly Equation, [arXiv:1107.0303 [hep-th]].

[9] N. Berkovits and C. Vafa, N=4 topological strings, Nucl. Phys. B433 (1995) 123 [hepth/9407190];

H. Ooguri and C. Vafa, All loop N=2 string amplitudes, Nucl. Phys. B451 (1995) 121 [hepth/9505183].

[10] I. Antoniadis, S. Hohenegger and K. S. Narain, N=4 Topological Amplitudes and String Effective Action, Nucl. Phys. B 771 (2007) 40 [hep-th/0610258];

I. Antoniadis and S. Hohenegger, Topological amplitudes and physical couplings in string theory, Nucl. Phys. Proc. Suppl. 171 (2007) 176 [hep-th/0701290].

[11] W. Lerche and S. Stieberger, 1/4 BPS states and nonperturbative couplings in N=4 string theories, Adv. Theor. Math. Phys. 3 (1999) 1539 [hep-th/9907133]. 
[12] A. Klemm, M. Kreuzer, E. Riegler and E. Scheidegger, Topological string amplitudes, complete intersection Calabi-Yau spaces and threshold corrections, JHEP 0505 (2005) 023 [hepth/0410018];

I. Antoniadis, E. Gava, K. S. Narain and T. R. Taylor, N=2 type II heterotic duality and higher derivative F terms, Nucl. Phys. B 455 (1995) 109 [hep-th/9507115].

[13] J. A. Harvey and G. W. Moore, Algebras, BPS states, and strings, Nucl. Phys. B 463 (1996) 315 [hep-th/9510182]. J. A. Harvey and G. W. Moore, On the algebras of BPS states, Commun. Math. Phys. 197 (1998) 489 [hep-th/9609017].

[14] M. R. Gaberdiel, S. Hohenegger and D. Persson, Borcherds Algebras and N=4 Topological Amplitudes, JHEP 1106 (2011) 125 [arXiv:1102.1821 [hep-th]];

S. Hohenegger and D. Persson, Enhanced Gauge Groups in N=4 Topological Amplitudes and Lorentzian Borcherds Algebras, Phys. Rev. D 84 (2011) 106007 [arXiv:1107.2301 [hep-th]].

[15] H. Ooguri, A. Strominger and C. Vafa, Black hole attractors and the topological string, Phys. Rev. D 70 (2004) 106007 [hep-th/0405146].

[16] A. Dabholkar, F. Denef, G.W. Moore and B. Pioline, Precision counting of small black holes, JHEP 0510 (2005) 096. [hep-th/0507014].

[17] A. Dabholkar, Exact counting of black hole microstates, Phys. Rev. Lett. 94 (2005) 241301 [hep-th/0409148].

[18] I. Antoniadis and S. Hohenegger, N=4 Topological Amplitudes and Black Hole Entropy, Nucl. Phys. B 837 (2010) 61 [arXiv:0910.5596 [hep-th]].

[19] T. Eguchi, H. Ooguri and Y. Tachikawa, Notes on the K3 Surface and the Mathieu group $M_{24}$, Exper. Math. 20 (2011) 91 [arXiv:1004.0956 [hep-th]].

[20] M. C. N. Cheng, K3 Surfaces, N=4 Dyons, and the Mathieu Group M24, Commun. Num. Theor. Phys. 4 (2010) 659 [arXiv:1005.5415 [hep-th]].

[21] M. R. Gaberdiel, S. Hohenegger and R. Volpato, Mathieu twining characters for K3, JHEP 1009 (2010) 058 [arXiv:1006.0221 [hep-th]].

[22] M. R. Gaberdiel, S. Hohenegger and R. Volpato, Mathieu Moonshine in the elliptic genus of K3, JHEP 1010 (2010) 062 [arXiv:1008.3778 [hep-th]].

[23] T. Eguchi and K. Hikami, Note on Twisted Elliptic Genus of K3 Surface, Phys. Lett. B 694 (2011) 446 [arXiv:1008.4924 [hep-th]].

[24] M. R. Gaberdiel, S. Hohenegger and R. Volpato, Symmetries of K3 sigma models, arXiv:1106.4315 [hep-th].

[25] S. Govindarajan, Brewing Moonshine for Mathieu, arXiv:1012.5732 [math.NT].

[26] S. Govindarajan, Unravelling Mathieu Moonshine, arXiv:1106.5715 [hep-th]. 
[27] A. Taormina and K. Wendland, The overarching finite symmetry group of Kummer surfaces in the Mathieu group $M_{2} 4$, arXiv:1107.3834 [hep-th].

[28] M. C. N. Cheng and J. F. R. Duncan, On Rademacher Sums, the Largest Mathieu Group, and the Holographic Modularity of Moonshine, arXiv:1110.3859 [math.RT].

[29] S. Govindarajan and K. Gopala Krishna, BKM Lie superalgebras from dyon spectra in $Z(N)$ CHL orbifolds for composite N, JHEP 1005 (2010) 014 [arXiv:0907.1410 [hep-th]].

[30] S. Govindarajan, BKM Lie superalgebras from counting twisted CHL dyons, JHEP 1105 (2011) 089 [arXiv:1006.3472 [hep-th]].

[31] A.N. Schellekens and N.P. Warner, Anomalies, Characters and Strings, Nucl. Phys. B287, 317 (1987).

[32] E. Witten, Elliptic Genera And Quantum Field Theory, Commun. Math. Phys. 109, 525 (1987).

[33] W. Lerche, B.E.W. Nilsson, A.N. Schellekens and N.P. Warner, Anomaly Cancelling Terms From The Elliptic Genus, Nucl. Phys. B299 (1988) 91.

[34] T. Kawai, Y. Yamada, and S.-K. Yang, Elliptic genera and $\mathscr{N}=2$ superconformal field theory, Nucl. Phys. B 414, 191 (1994) [hep-th/9306096].

[35] M. Eichler and D. Zagier, The Theory of Jacobi Forms, Birkhäuser (1985).

[36] T. Eguchi and K. Hikami, Superconformal algebras and Mock Theta Functions 2. Rademacher expansion for K3 surface, Commun. Number Theory and Physics 3, 531 (2009) [arXiv:0904.0911 [math-ph]].

[37] T. Eguchi, H. Ooguri, A. Taormina and S.K. Yang, Superconformal algebras and string compactification on manifolds with SU(N) holonomy, Nucl. Phys. B 315, 193 (1989).

[38] S. P. Zwegers, Mock Theta Functions, Ph.D. thesis, Universiteit Utrecht (2002).

[39] T. Gannon, Moonshine beyond the Monster: The Bridge connecting Algebra, Modular Forms and Physics, Cambridge University Press (2006).

[40] J.G. Thompson, Some numerology between the Fischer-Griess Monster and the elliptic modular function, Bull. Lond. Math.Sंoc. 11 (1979) 352-3.

[41] S. Mukai, Finite groups of automorphisms of K3 surfaces and the Mathieu group, Invent. Math. 94 (1988) 183. S. Kondo, Niemeier lattices, Mathieu groups and finite groups of symplectic automorphisms of K3 surfaces, Duke Math. Journal 92 (1998) 593, appendix by S. Mukai.

[42] P.S. Aspinwall, K3 surfaces and string duality, In *Yau, S.T. (ed.): Differential geometry inspired by string theory* 1-95 [hep-th/9611137]. 
[43] W. Nahm and K. Wendland, A Hiker's guide to K3: Aspects of $N=(4,4)$ superconformal field theory with central charge $c=6$, Commun. Math. Phys. 216 (2001) 85 [hep-th/9912067].

[44] S. Hohenegger and S. Stieberger, BPS Saturated String Amplitudes: K3 Elliptic Genus and Igusa Cusp Form, Nucl. Phys. B 856 (2012) 413 [arXiv:1108.0323 [hep-th]].

[45] L.J. Dixon, V. Kaplunovsky and J. Louis, Moduli dependence of string loop corrections to gauge coupling constants, Nucl. Phys. B 355 (1991) 649.

[46] R.E. Borcherds, Automorphic forms with singularities on Grassmannians, Invent. Math. 132 (1998) 491.

[47] K. Foerger and S. Stieberger, Higher derivative couplings and heterotic type I duality in eightdimensions, Nucl. Phys. B559 (1999) 277 [hep-th/9901020]; String amplitudes and N=2, $d$ $=4$ prepotential in heterotic K3 $\times T^{2}$ compactifications, Nucl. Phys. B514 (1998) 135 [hepth/9709004].

[48] T. Kawai, $N=2$ heterotic string threshold correction, $K 3$ surface and generalized Kac-Moody superalgebra, Phys. Lett. B372 (1996) 59 [hep-th/9512046].

[49] G.W. Moore, Modular Forms And Two Loop String Physics, Phys. Lett. B176 (1986) 369.

[50] P. Mayr and S. Stieberger, Moduli dependence of one loop gauge couplings in $(0,2)$ compactifications, Phys. Lett. B355 (1995) 107 [hep-th/9504129].

[51] R. Dijkgraaf, E.P. Verlinde and H.L. Verlinde, Counting dyons in N=4 string theory, Nucl. Phys. B484 543 (1997) [hep-th/9607026].

[52] T. Eguchi and K. Hikami, Twisted Elliptic Genus for K3 and Borcherds Product, arXiv:1112.5928 [hep-th].

[53] R.E. Borcherds, Vertex algebras, Kac-Moody algebras, and the Monster, Proc. Natl. Acad. Sci. USA 83 (1986) 3068-71.

[54] I. Frenkel, J. Lepowsky, A.-Meurman, A natural representation of the Fischer-Griess monster with the modular function J as character, Proc. Natl. Acad. Sci. USA 81 (1984) 3256-60. 